\title{
A lei de Poiseuille como motivadora para o ensino de Cálculo
}

\author{
Gabriel Fernandez Ferrari Melo (D) Juscimar da Silva Araujo (D)
}

\section{Resumo}

Neste artigo, apresentamos a lei de Poiseuille, revisitada para o ensino de Cálculo Diferencial e Integral (CDI). Alguns estudos evidenciam a necessidade do uso de práticas alternativas para o ensino e a aprendizagem do CDI, visando dirimir a evasão e o alto índice de reprovação que há nessa disciplina. Assim, utilizamos uma abordagem baseada na Modelagem Matemática, em que, por meio da modelagem e descrição da circulação sanguínea, obstrução e ramificação dos capilares, apresentamos os principais conceitos referentes ao CDI, a saber: o estudo de funções, limites, derivadas e integrais; evidenciando como esses podem ser empregados em sala de aula; apresentando assim a conexão entre a Matemática e o mundo real, de modo a contribuir para dirimir os problemas que se percebem em relação ao ensino e à aprendizagem nessa disciplina; além de possibilitar um lugar na Matemática para o espírito criativo e investigativo.

Palavras-chave: Ensino de Cálculo; Aplicações da Matemática; Lei de Poiseulle; Modelagem Matemática.

\begin{abstract}
In this article, we present Poiseuille's law, revisited for teaching Differential and Integral Calculus (CDI). Some studies show the need to use alternative practices for teaching and learning the CDI, aiming to reduce the dropout rate and the high failure rate in this discipline. Thus, we use an approach based on Mathematical Modeling, in which, through the modeling and description of blood circulation, obstruction and branching of capillaries, we present the main concepts related to the ICD, namely: the study of functions, limits, derivatives and integrals; showing how these can be used in the classroom; thus presenting the connection between Mathematics and the real world, in order to contribute to resolve the problems that are perceived in relation to teaching and learning in this subject; in addition to providing a place in Mathematics for the creative and investigative spirit.
\end{abstract}

Keywords: Calculus teaching; Applications of Mathematics; Poiseulle's Law; Mathematical Modeling.

\section{Introdução}

A origem do Cálculo Diferencial e Integral (CDI), de acordo com [3], está fortemente ligada ao século XVII e a dois importantes nomes da ciência: o do inglês Isaac Newton (1642 - 1727), sendo 
amplamente reconhecido como um dos cientistas mais influentes de todos os tempos, e o do alemão Goltfried Wilhelm Leibniz (1646 - 1716), um polímata que é figura importante na história da Ciência, em especial, da Matemática.

O CDI está presente e é um importante componente curricular nos cursos de graduação em Matemática, Física, Engenharias, entre outras na área das ciências exatas. É nessa componente que os alunos têm um contato amplo e formal com alguns conceitos importantes, tais como os de limite, derivada e integrais; conceitos esses que proporcionam uma base sólida para o estudo, por exemplo, da Matemática no ensino superior e suas aplicações em situações do mundo real.

Nesse âmbito ressaltamos que as disciplinas de Cálculo, de modo geral, têm se mostrado desafiadoras para boa parte dos acadêmicos nos cursos de ciências exatas, como colocado por [1, 11]. Desse modo, entendemos que, ao optarmos por diferentes metodologias de ensino de CDI, pode-se dirimir os entraves no ensino e na aprendizagem dessa área do conhecimento matemático.

Seguindo esse viés, ressaltamos que em tempo atual, no âmbito da Educação Matemática, encontramos três tendências muito difundidas nos últimos tempos, quais sejam: a introdução de aspectos de aplicações, resolução de problemas e a modelagem matemática. Nesse contexto, abordaremos no presente artigo essa última, por entendermos que ao trabalhar com a modelagem matemática na sala de aula podemos provocar sua associação com questões reais, o reconhecimento de problemas e o levantamento de hipóteses.

De posse dessa tendência, revisitaremos a lei de Poiseuille; enquanto realizaremos sua modelagem a partir da situação fundamental que é o alicerce para seu desenvolvimento: o escoamento laminar até sua formulação e determinação do fluxo sanguíneo em um capilar. Além disso, apresentaremos modelos que estendem essa lei, mostrando sua aplicabilidade para o estudo da obstrução e ramificação de vasos sanguíneos, onde evidenciaremos os aspectos fundamentais do CDI presente nessa modelagem, além de explicitar como os mesmos podem ser utilizados como ferramenta motivadora e alternativa para o ensino dessa disciplina.

Dessa forma, exploramos o uso da lei de Poiseuille, diferindo de como ela é comumente apresentada em textos, a exemplo de $[4,8]$. Entendemos que ao estabelecer forte conexão entre a realidade e a Matemática, cria-se um espaço para discussão, criatividade e investigação em sala de aula.

\section{Algumas considerações sobre Modelagem Matemática e fenômenos do mundo real}

No presente artigo buscaremos revisitar a Lei de Poiseuille como uma alternativa didática para o ensino do CDI via Modelagem Matemática ${ }^{1}$. Dessa forma, torna-se importante destacarmos os aspectos teóricos que fundamentam a modelagem, para explicitar alguns tipos de modelos, bem como os passos para que esses sejam concebidos, testados e aperfeiçoados, e sua utilização para o ensino de Matemática.

A modelagem tem sua base fundada na matemática aplicada e centra-se no desenvolvimento de um modelo matemático, ou simplesmente modelo, o qual pode ser apresentado como uma representação de um sistema real [5]. Todavia, o desenvolvimento de um modelo, isto é, o ato da modelagem, dá-se através de fundamentos de um processo, os quais devem ser seguidos para obtermos sistematizadamente nosso modelo. De início, apresentaremos as diferenças entre os tipos fundamentais de modelos conforme colocado em [5]: modelos empíricos e teóricos.

Os modelos ditos empíricos têm, como ponto de partida, um processo sistemático de medição.

\footnotetext{
${ }^{1}$ Para fins textuais, utilizaremos o termo Modelagem para nos referirmos à Modelagem Matemática.
} 
Isto é, os mesmos baseiam-se na averiguação experimental, em que por meio da análise dos dados obtidos formula-se uma equação ou sistema de equações que vinculam as grandezas de interesse envolvidas no fenômeno estudado. Nesse âmbito, citamos, por exemplo, o modelo obtido para a lei do decaimento radioativo, a qual fora definido através da medição do número de partículas emitidas pela amostra emissora por unidade de tempo, usando um "contador Geiger" para tal aferição e a partir disso identificando, através de uma curva de ajuste, o comportamento exponencial desse fenômeno.

Já os modelos teóricos são fundamentados em algumas etapas, conforme enunciamos abaixo:

- Todo modelo teórico é obtido em relação a um determinado contexto, em que se ajustam as "hipóteses de trabalho", isto é, as condições e leis compatíveis com as restrições da situação estudada;

- Todo novo modelo obtido deve ser submetido a testes de confiabilidade, de modo a concordar, ou não, com os resultados previamente encontrados sobre a realidade objetiva;

- Um modelo aperfeiçoado, isto é, com menos restrições ou menos hipóteses de trabalho deve reduzir-se aos casos mais simples já determinados nas respectivas situações limites;

- Modelos mais simples não devem ser descartados, pois podem resolver de forma simples problemas em determinadas condições.

Os tópicos mencionados acima formam os fundamentos da modelagem como colocado por [5].

Em relação ao presente texto, destacamos que trabalharemos tanto com modelos empíricos quanto teóricos; visto que as leis fundamentais que estabelecem as primeiras condições para a modelagem da lei de Poiseuille advêm diretamente da experimentação. Nesse caso apresentaremos uma discussão qualitativa dos resultados, bem como os mesmos apresentam-se matematicamente. Posteriormente, passaremos a nos ater aos modelos teóricos, os quais serão desenvolvidos sob as hipóteses experimentais, mas valendo-se de todos os fundamentos da modelagem já citados.

\section{Apresentando a lei de Poiseuille}

Um dos pontos de interesse no estudo da dinâmica de fluidos é entender como os estados de posição comportam-se com a evolução temporal do escoamento. Todavia, diferentemente do estudo da dinâmica de partículas e trajetórias de outros corpos, os fluidos constituem sistemas contínuos, que não podem ser tratados de forma discretizada, tornando a análise de sua dinâmica mais complexa de ser concebida, seja analiticamente ou por métodos numéricos.

Tendo isso em vista, torna-se interessante para tal estudo a avaliação de aspectos fundamentais do fluido, de modo que seja possível estabelecer uma descrição do seu comportamento sob condições particulares que se relacionam com sua natureza física.

Nesse contexto, citamos a lei de Poiseuille, publicada em 1840/1841 por Jean Leonard Marie Poiseuille $(1797$ - 1869), um físico e médico francês. Essa lei apresentou importantes contribuições para a hemodinâmica - a qual é um conjunto de componentes físicos que constituem o bombeamento de sangue no sistema cardiovascular - visto que permite a descrição do fluxo sanguíneo em vasos cilíndricos como colocado por [9], a qual é dada pela Equação (1)

$$
\mathrm{V}=\frac{\pi \Delta \mathrm{P}}{8 \eta \mathrm{l}} \mathrm{R}^{4},
$$


em que $\Delta \mathrm{P}$ é a diferença de pressões, $\eta$ é uma constante chamada de coeficiente de viscosidade, l e R são, respectivamente, o comprimento e o raio do vaso cilíndrico. No entanto, a formulação obtida na lei de Poiseuille mostra-se capaz de modelar não apenas o fluxo do sangue, mas também uma classe de fluidos sob algumas hipóteses, que são:

- O fluido tem de ser viscoso;

- O fluido deve obedecer ao escoamento laminar;

- O vaso cilíndrico tem que possuir mais de $0.4 \mathrm{~mm}$ de diâmetro.

Partindo dessas hipóteses, revisitaremos a lei de Poiseuille, de modo que, através da ótica da modelagem, nas subseções seguintes abordaremos uma descrição dos aspectos físicos e matemáticos relacionados à Equação (1) como uma alternativa didática ao ensino de CDI, evidenciando como os principais conceitos dessa disciplina podem ser empregados na descrição dessa lei, além de mostrarmos como os aspectos matemáticos presentes na estrutura curricular do CDI levam-nos a obter importantes considerações do modelo trabalhado com a finalidade de contextualizar e motivar o ensino dessa disciplina.

\subsection{Viscosidade e escoamento laminar}

De início, começaremos revisitando as hipóteses de trabalho da lei de Poiseuille, em especial as que se referem à classe de fluidos por ela trabalhada: os fluidos viscosos. Essa propriedade é da natureza física do fluido, isto é, relaciona-se a sua constituição ao nível molecular, mas seus efeitos são notados em escala macroscópica. Não obstante, sua presença é mais bem perceptível quando observamos o deslizamento de camadas fluidas umas sobre as outras, dando origem a tensões tangenciais. Para melhor entendermos esse fenômeno, faremos a descrição do resultado empírico do que ocorre quando puxamos uma placa de uma caixa com área lateral A com uma força $\overrightarrow{\mathrm{F}}$ na direção do eixo x, a qual está sobre um recipiente com um fluido viscoso, como o ilustrado na Figura 1.

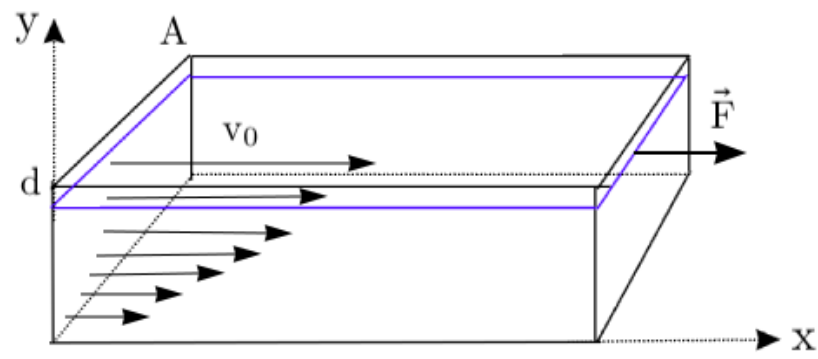

Figura 1: Esquematização do escoamento de um fluido viscoso em uma caixa retangular.

A força $\vec{F}$ aplicada gera um deslocamento na direção do eixo x, todavia, o fluido não se desloca de forma homogênea, mas como se fosse composto por uma superposição de finas lâminas em que a medida que temos uma maior proximidade com o fundo do recipiente temos uma diminuição da velocidade $\mathrm{v}_{\mathrm{o}}$, e, exatamente, no fundo, temos que o fluido permanece em repouso. Esse fato 
experimental, permite o início do processo de modelagem para o escoamento de um fluido viscoso, visto que temos uma relação de proporcionalidade entre a velocidade e a profundidade da caixa, que é linear, mas inversamente proporcional. Com isso, podemos estabelecer uma função para a velocidade do deslizamento em termos de uma altura qualquer y, sendo essa: v : [0,d] $\rightarrow \mathbb{R}$ com lei de associação dada pela Função (2)

$$
\mathrm{v}(\mathrm{y})=\frac{\mathrm{v}_{0}}{\mathrm{~d}} \mathrm{y}
$$

Em que, para $\mathrm{y}=0$ temos a situação de repouso do fluido e para $\mathrm{y}=\mathrm{d}$ temos a velocidade $\mathrm{v}_{0}$ da parte superior do recipiente, evidenciando que essa equação contém as informações referentes ao nosso modelo para os níveis de altura avaliados pela caixa da Figura 1.

A Equação (2) fornece-nos uma descrição da velocidade em qualquer altura y, porém ainda precisamos de um modelo para o entendimento das tensões tangenciais, as quais permitem que se mantenha o deslocamento da placa superior com velocidade $\mathrm{v}_{0}$, . Para tanto utilizaremos a lei de Newton da viscosidade, que se expressa pela Equação (3)

$$
\frac{\mathrm{F}}{\mathrm{A}}=\eta \frac{\mathrm{v}_{0}}{\mathrm{~d}}=\eta \frac{\Delta \mathrm{v}}{\Delta \mathrm{y}}=\eta \frac{\mathrm{dv}}{\mathrm{dy}}
$$

Isto é, a tensão tangencial $\left(\frac{\mathrm{F}}{\mathrm{A}}\right)$ é proporcional à variação da velocidade v em relação à altura, o que entra em consonância com o modelo empírico que tratamos acima, visto que à medida que temos uma menor variação da altura no eixo y temos uma maior resistência ao escoamento que ocorre nas proximidades do nível zero do modelo tratado. Ademais, é importante ressaltar a presença do termo $\eta$, o qual é uma constante de proporcionalidade chamada coeficiente de viscosidade do fluido; em especial quanto maior o valor numérico de $\eta$ mais espesso e maior resistência ao escoamento o fluido possui.

Além disso, é impar que especifiquemos os aspectos matemáticos trabalhados nessa primeira descrição do modelo estudado. Em especial, nesse primeiro momento evidenciamos a relevância da Equação (1), a qual nos mostra que a descrição do escoamento laminar, o qual é base para a lei de Poiseuille, pode ser tratada em termos das variações da velocidade, sem uso explícito das derivadas, o que possibilita sua apresentação aos discentes desde o início do curso de CDI.

\subsection{Escoamento viscoso em um tubo cilíndrico}

A partir do entendimento do escoamento laminar, bem como dos efeitos da viscosidade na dinâmica do fluido, podemos nos direcionar para a hipótese do escoamento do fluido em um cilindro. Para tanto, esquematizaremos esse corpo através da Figura 2 e suporemos o escoamento de um fluido viscoso que parte do ponto $\mathrm{A}$ até o ponto $\mathrm{B}$, atravessando um cilindro de comprimento $\mathrm{l}$ e raio $\mathrm{R}$. Não obstante, iremos, também, descrever um cilindro de raio $\mathrm{r}^{\prime}$ interno ao cilindro maior. 


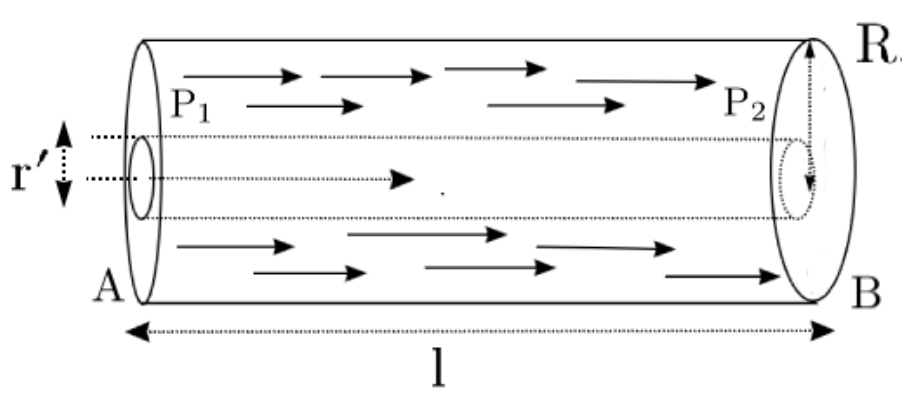

Figura 2: Esquematização do escoamento de um fluido viscoso em um corpo cilíndrico.

Dada tais considerações, realizaremos uma descrição do fluxo do escoamento em termos de uma variável $\mathrm{r}$ que representa um raio arbitrário definido de maneira consoante ao esquema proposto pela Figura 2, isto é $\mathrm{r} \in\left[\mathrm{r}^{\prime}, \mathrm{R}\right]$. Para isso, usaremos a lei da viscosidade definida na Equação (3) em que as pressões serão descritas pela relação da pressão hidrostática: $P_{i}=\frac{F_{i}}{A_{i}} \operatorname{com~} i=1,2$. É importante pontuarmos que a força relacionada à pressão $\mathrm{P}_{1}$ será positiva, enquanto a força em relação à pressão $\mathrm{P}_{2}$ será descrita com sinal negativo. Tal consideração é efetuada para modelarmos em que sentido as forças são aplicadas. Aqui consideramos o sentido positivo dado pelo fluxo do fluido. Em relação às áreas $A_{i}$ tomaremos a área da secção circular do cilindro expressa pelo raio $\mathrm{r}$ já definido. Com isso, podemos escrever a força resultante $\mathrm{F}_{\mathrm{r}}$ atuante no sistema dada por:

$$
\mathrm{F}=\mathrm{F}_{1}+\mathrm{F}_{2}=\mathrm{P}_{1} \pi \mathrm{r}^{2}-\mathrm{P}_{2} \pi \mathrm{r}^{2}=\left(\mathrm{P}_{1}-\mathrm{P}_{2}\right) \pi \mathrm{r}^{2}
$$

Agora, estamos prontos para expressarmos a lei da viscosidade para nosso modelo em um corpo de simetria cilíndrica. Assim, relacionaremos a parte dos termos diferenciais da Equação (3) e a Equação (4) tomando uma área $\mathrm{A}$ dada por $\mathrm{A}=2 \pi \mathrm{rl}$ e a função da velocidade de escoamento será definida por $\mathrm{v}:\left[\mathrm{r}^{\prime}, \mathrm{R}\right] \rightarrow \mathbb{R}$. Ademais, é necessário realizarmos uma ressalva, a qual tomaremos que $\frac{\mathrm{dv}}{\mathrm{dr}}<0$, que decorre do caráter de que à medida que o raio r varia, temos uma diminuição da velocidade por estarmos trabalhando sob a hipótese do escoamento laminar. Com efeito, podemos prosseguir para o seguinte desenvolvimento algébrico:

$$
\begin{array}{r}
\frac{\mathrm{F}}{\mathrm{A}}=\frac{\left(\mathrm{P}_{1}-\mathrm{P}_{2}\right) \pi \mathrm{r}^{2}}{2 \pi \mathrm{rl}}=-\eta \frac{\mathrm{dv}}{\mathrm{dr}} \\
\frac{\left(\mathrm{P}_{1}-\mathrm{P}_{2}\right) \mathrm{r}}{2 \mathrm{l}}=-\eta \frac{\mathrm{dv}}{\mathrm{dr}} \\
\therefore \frac{\mathrm{dv}}{\mathrm{dr}}=-\frac{\left(\mathrm{P}_{1}-\mathrm{P}_{2}\right)}{2 \eta \mathrm{l}} \mathrm{r} .
\end{array}
$$

A Equação (5) apresenta a velocidade v sendo dada por um termo diferencial em relação à variável independente r. Ademais, essa é uma Equação Diferencial Ordinária (E.D.O). Em suas classificações a mesma é dita de variáveis separáveis, no entanto, no enfoque do nosso trabalho não se objetiva o tratamento da equação (5) com o rigor exigido para uma E.D.O. Entretanto, a dedução dessa Equação mostra uma primeira motivação aos estudantes, ao passo que apresenta a noção de derivada como elemento ímpar na modelagem e descrição de sistemas físicos. 
Por outro lado, ao termos uma E.D.O, torna imperativo enunciar o Teorema Fundamental do Cálculo, dado que esse apresenta-se como uma poderosa ferramenta para explicitarmos a função $\mathrm{v}(\mathrm{r})$, visto a possibilidade de relacionar os conceitos de derivada e integral (Parte 1), bem como o cálculo de integrais definidas (Parte 2).

Teorema 1. (Teorema Fundamental do Cálculo - Parte 1) Seja f : [a,b] $\rightarrow \mathbb{R}$ uma função contínua. A função $\mathrm{F}:[\mathrm{a}, \mathrm{b}] \rightarrow \mathbb{R}$ definida pela expressão

$$
F(x)=\int_{a}^{x} f(s) d s
$$

é derivável e $\mathrm{F}^{\prime}(\mathrm{x})=\mathrm{f}(\mathrm{x})$ para todo $\mathrm{x} \in(\mathrm{a}, \mathrm{b})$.

Demonstração. Ver referência [10].

Teorema 2. (Teorema Fundamental do Cálculo - Parte 2). Dada uma função contínua $\mathrm{f}[\mathrm{a}, \mathrm{b}] \rightarrow \mathbb{R}$ e uma de suas primitivas $\mathrm{G}$, então

$$
\int_{a}^{b} f(x) d x=G(b)-G(a) .
$$

Demonstração. Ver referência [6].

Sabendo que a função v(r) é contínua, por implicações físicas do movimento, o que satisfaz as hipóteses do Teorema Fundamental do Cálculo, assim possibilitando que possamos nos valer do mesmo, em especial a Parte 2, em que tomaremos $\mathrm{a}=\mathrm{r}$ e $\mathrm{b}=\mathrm{R}$. Além disso, destacamos que faremos uma mudança de variáveis na integração para s, assim como colocado pelo Teorema 1, de modo que, após a integração da Função (5), obtenhamos uma função da velocidade em termos da variável $\mathrm{r}$ definida no intervalo $\left[\mathrm{r}^{\prime}, \mathrm{R}\right]$. Efetuada essas considerações, realizaremos a integração dessa Função, que nos leva ao seguinte desenvolvimento,

$$
\begin{aligned}
\int_{\mathrm{r}}^{\mathrm{R}} \frac{\mathrm{dv}}{\mathrm{dr}} \mathrm{dr} & =-\int_{\mathrm{r}}^{\mathrm{R}} \frac{\left(\mathrm{P}_{1}-\mathrm{P}_{2}\right)}{2 \eta \mathrm{l}} \mathrm{sds} \\
\mathrm{v}(\mathrm{R})-\mathrm{v}(\mathrm{r}) & =-\left[\frac{\left(\mathrm{P}_{1}-\mathrm{P}_{2}\right)}{\eta \mathrm{l}} \mathrm{s}^{2}\right]_{\mathrm{r}}^{\mathrm{R}} \\
\mathrm{v}(\mathrm{r}) & =\frac{\left(\mathrm{P}_{1}-\mathrm{P}_{2}\right)}{4 \eta \mathrm{l}}\left(\mathrm{R}^{2}-\mathrm{r}^{2}\right) .
\end{aligned}
$$

Nele, o termo v $(R)=0$, pois a velocidade anula-se nas paredes do cilindro. Ademais, a Equação (8) dá-nos a velocidade (v) em relação a um raio arbitrário r que condiz com as hipóteses de trabalho para o modelo. Não só isso, mas a descrição da velocidade dá-se por uma função do segundo grau, mostrando que a velocidade possui um caráter parabólico. Além disso, a obtenção da Equação (8) torna-se de imensa valia para o estudo desse modelo, visto que, ao interpretamos seus aspectos matemáticos, possibilitamos um estudo de funções que se conecta com o sentido real do sistema que estamos modelando.

Primeiramente, analisaremos as raízes dessa equação, as quais podem ser vistas à medida que decompomos o termo $\left(R^{2}-r^{2}\right)$ em $(R+r)(R-r)$ evidenciando que as raízes são obtidas para 
$r= \pm R$. No entanto, como sabemos que a velocidade $v$ está definida para valores de $r \in\left[r^{\prime}, R\right]$ ou seja, o ponto -R não pertence ao domínio da função, é interessante, entretanto, discutirmos o aparecimento desse valor como raiz da Equação (8). O aparecimento do sinal negativo relaciona-se diretamente com a simetria do corpo estudado e do perfil da velocidade: parabólico, pois a parte que diz respeito a $-\mathrm{R}$ pode ser entendida de forma análoga ao $\mathrm{R}$ positivo dado que esse é apenas uma reflexão do mesmo.

Agora, apresentaremos a concordância da Equação (8) com o modelo do escoamento laminar proposto na subseção anterior, em especial pelo ilustrado na Figura 1, por isso plotamos o gráfico da função (8) na Figura 3 (a) e apresentamos na Figura 3-(b) traçamos o comportamento das velocidades, de fato, ao se aproximarem dos extremos do cilindro, isto é quando $\mathrm{r} \rightarrow|\mathrm{R}|$, tendem a zero.

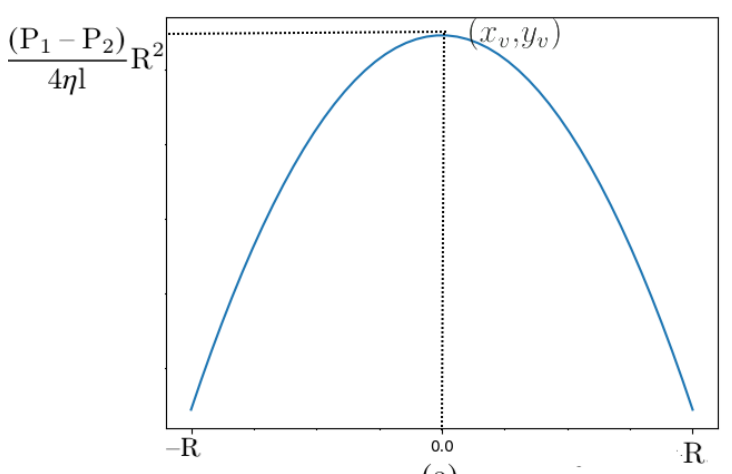

(a)

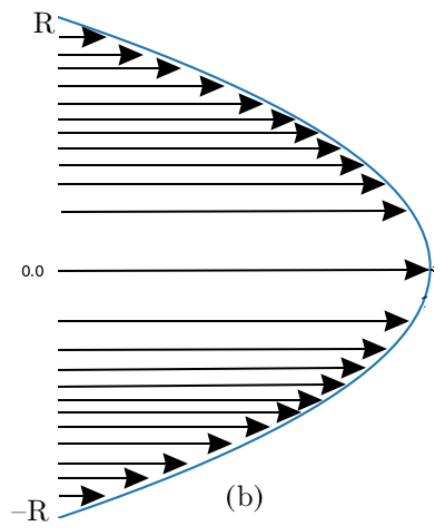

(b)

Figura 3: Em (a) gráfico da função (8) e em (b) o perfil parabólico da velocidade, que ilustra o escoamento laminar.

Em especial, a interpretação das raízes da equação da velocidade bem como a forma parabólica dessa equação compactuam com o modelo previamente apresentado. Além disso, a modelagem e dedução da equação (5) motiva e possibilita a inserção de um dos conceitos substanciais do CDI: O teorema Fundamental do Cálculo.

\subsection{Taxa de variação e gradiente da velocidade}

Nessa subseção resgataremos uma equação previamente trabalhada: A Função (5). Essa, que fora obtida ao aplicarmos as condições do escoamento laminar para um corpo com simetria cilíndrica também nos permite obter informações acerca da dinâmica do fluido. Em especial, tomares $\alpha(\mathrm{r})=$ $\frac{\mathrm{dv}}{\mathrm{dr}}$ e assim temos a expressão dada por (9)

$$
\alpha(\mathrm{r})=\frac{\mathrm{P}_{1}-\mathrm{P}_{2}}{2 \eta \mathrm{l}} \mathrm{r} .
$$

Nesse ponto é interessante analisarmos a função $\alpha(\mathrm{r})$, de modo a entendermos o seu sentido no presente contexto da modelagem da lei de Poiseuille. Para tanto, iremos nos valer da definição 1 conforme colocado por [8]. 
Definição 1. Definimos a aceleração instantânea a(t) num instante de tempo t como o limite das velocidades médias num intervalo $[\mathrm{t}, \mathrm{t}+\mathrm{h}]$, isto é:

$$
a(t)=\lim _{h \rightarrow 0} \frac{v(t+h)-v(t)}{h}=\frac{d v}{d t} .
$$

A Definição 1 estabelece que a aceleração a(t) é a derivada temporal da velocidade. No entanto, podemos trabalhar de forma análoga para a função (9), em que definiremos uma aceleração em relação à variável $\mathrm{r}$, a qual conceituaremos por aceleração radial $\alpha(\mathrm{r})$. Dessa forma, teremos a possibilidade de mensurar a variação da velocidade em termos da distância $r$ do cilindro apresentado na Figura 2, a qual possui comportamento descrito pelo gráfico da Figura 4.

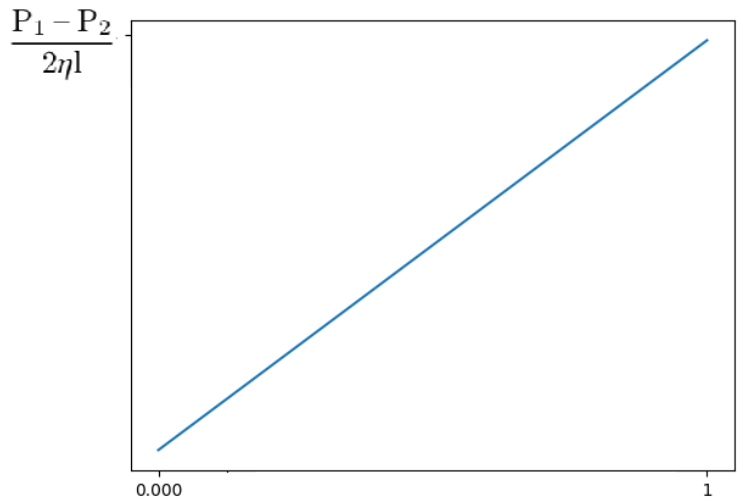

Figura 4: Gráfico da função (9); foram usados valores arbitrários para o termo $\frac{\mathrm{P}_{1}-\mathrm{P}_{2}}{2 \eta \mathrm{l}}$.

Com isso explicitado, agora estudaremos essa aceleração. De início, é interessante notarmos que o caráter linear presente na Equação (9) mostra uma concordância com a Função (2) do modelo do escoamento viscoso. Além disso, à medida que temos uma aceleração capaz de variar ao longo de sua variável, temos aqui um movimento que difere dos apresentados como motivadores do estudo da cinemática no CDI, visto que, comumente têm-se exemplos para os quais a aceleração apresentase como uma constante. Dito isso, temos que essa conceituação apresenta-se como uma forma alternativa aos tradicionais exemplos de aplicações cinemáticas tratadas no âmbito do CDI.

\subsection{Fluxo sanguíneo e vazão}

Nessa subseção determinaremos o fluxo sanguíneo ao longo de um capilar, isto é, o volume de sangue por unidade de tempo: o qual é a lei de Poiseuille. Para tanto, partiremos do modelo já descrito na Figura 2, de modo a determinarmos o volume de sangue que flui em uma secção transversal do capilar.

Assim, consideraremos a Figura 5-(a), que esquematiza a secção circular de interesse em que o conjunto de raios menores $\mathrm{r}_{1}, \mathrm{r}_{2}, \ldots, \mathrm{r}_{\mathrm{n}}$ estão igualmente espaçados de modo que possamos determinar a área para esse anel circular, área que é dada pela Equação (11), 


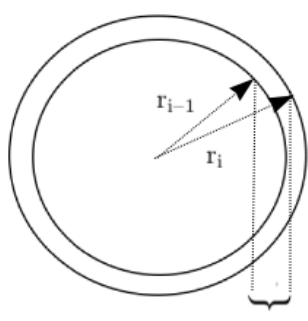

(a)

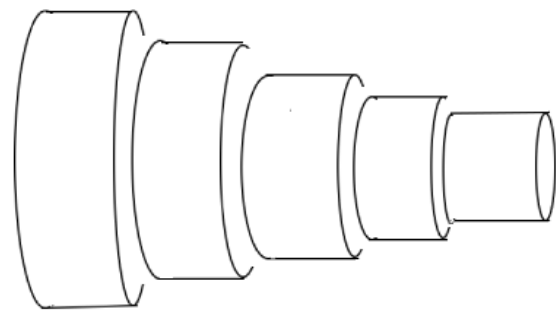

(b)

Figura 5: Esquematização da secção circular do cilindro em (a) e dos vasos concêntricos em (b) da Figura 2.

$$
\begin{aligned}
\mathrm{A}_{\mathrm{r}_{\mathrm{i}}}-\mathrm{A}_{\mathrm{r}_{\mathrm{i}-1}} & =\pi \mathrm{r}_{\mathrm{i}}^{2}-\pi \mathrm{r}_{\mathrm{i}-1}^{2} \\
& =\pi\left(\mathrm{r}_{\mathrm{i}}+\mathrm{r}_{\mathrm{i}-1}\right)\left(\mathrm{r}_{\mathrm{i}}-\mathrm{r}_{\mathrm{i}-1}\right) \\
& \approx 2 \pi \mathrm{r}_{\mathrm{i}} \Delta \mathrm{r}_{\mathrm{i}}
\end{aligned}
$$

em que $\Delta r_{i}=r_{i}-r_{i-1}$, e realizamos a aproximação de que $r_{i}=r_{i-1}$ pelo fato de igual espaçamento entre os raios; aqui explicitamos o índice i em $\Delta \mathrm{r}_{\mathrm{i}}$ para melhor visualização da diferença dos raios.

Com a determinação da área do anel circular podemos então expressar a quantidade de sangue que passa por ele. Visto que estamos trabalhando com a hipótese de que o capilar é pequeno podemos considerar que a velocidade é praticamente constante e dada pela Equação (8); dessa forma o volume $\Delta \mathrm{v}_{\mathrm{i}}$ no anel é dado pela Equação (12)

$$
\Delta \mathrm{v}_{\mathrm{i}}=\left(2 \pi \mathrm{r}_{\mathrm{i}} \Delta \mathrm{r}_{\mathrm{i}}\right) \cdot \frac{\left(\mathrm{P}_{1}-\mathrm{P}_{2}\right)}{4 \eta \mathrm{l}}\left(\mathrm{R}^{2}-\mathrm{r}_{\mathrm{i}}^{2}\right) .
$$

Não obstante, podemos estender essa modelagem para os diversos vasos concêntricos do capilar como apresentado na Figura 5-(b), uma vez que, a extensão desse modelo garante a quantidade aproximada de volume total $(\Delta \mathrm{V})$ em todo o capilar, como feito pela Equação (13)

$$
\Delta \mathrm{V}=\sum_{\mathrm{i}=1}^{\mathrm{n}}\left[\left(2 \pi \mathrm{r}_{\mathrm{i}} \Delta \mathrm{r}_{\mathrm{i}}\right) \cdot \frac{\left(\mathrm{P}_{1}-\mathrm{P}_{2}\right)}{4 \eta \mathrm{l}}\left(\mathrm{R}^{2}-\mathrm{r}_{\mathrm{i}}^{2}\right)\right] .
$$

Partindo da Equação (13), podemos então avaliar o caso para $\mathrm{n} \rightarrow \infty$ o qual garante uma melhor aproximação para o fluxo sanguíneo $(\mathrm{V}(\mathrm{r})$ ), uma vez que o mesmo tende para a integral apresentada em (14)

$$
\begin{aligned}
\mathrm{V}(\mathrm{r}) & =\lim _{\mathrm{n} \rightarrow \infty} \sum_{\mathrm{i}=1}^{\mathrm{n}}\left[\left(2 \pi \mathrm{r}_{\mathrm{i}} \Delta \mathrm{r}_{\mathrm{i}}\right) \cdot \frac{\left(\mathrm{P}_{1}-\mathrm{P}_{2}\right)}{4 \eta \mathrm{l}}\left(\mathrm{R}^{2}-\mathrm{r}_{\mathrm{i}}^{2}\right)\right] \\
& =\int_{0}^{\mathrm{r}} \frac{\left(\mathrm{P}_{1}-\mathrm{P}_{2}\right) \pi}{2 \eta \mathrm{l}}\left(\mathrm{R}^{2} \mathrm{~s}-\mathrm{s}^{3}\right) \mathrm{ds}
\end{aligned}
$$


- onde alteramos a função de integração para s como explicitado no teorema 1 e tomamos os extremos de integração no intervalo [0,r], pois consideramos o eixo do tubo cilíndrico como origem das coordenadas. Assim, podemos, prosseguir no desenvolvimento da integral de modo,

$$
\begin{aligned}
\mathrm{V}(\mathrm{r}) & =\int_{0}^{\mathrm{r}} \frac{\left(\mathrm{P}_{1}-\mathrm{P}_{2}\right) \pi}{2 \eta \mathrm{l}}\left(\mathrm{R}^{2} \mathrm{~s}-\mathrm{s}^{3}\right) \mathrm{ds} \\
& =\frac{\left(\mathrm{P}_{1}-\mathrm{P}_{2}\right) \pi}{2 \eta \mathrm{l}}\left[\mathrm{R}^{2} \frac{\mathrm{s}^{2}}{2}-\frac{\mathrm{s}^{4}}{4}\right]_{0}^{\mathrm{r}} \\
& =\frac{\left(\mathrm{P}_{1}-\mathrm{P}_{2}\right) \pi}{2 \eta \mathrm{l}}\left[\mathrm{R}^{2} \frac{\mathrm{r}^{2}}{2}-\frac{\mathrm{r}^{4}}{4}\right]
\end{aligned}
$$

A função (15) permite uma descrição da vazão para o escoamento do cilindro com um raio arbitrário r, de modo que possamos então ajustá-la para os devidos fins de interesse, na descrição de outros sistemas. Todavia, torna-se ainda interessante estudarmos essa função à medida que o raio $\mathrm{r}$ aproxima-se do limite do nosso sistema, isto é, suficiente próximo do raio $\mathrm{R}$, o que é feito tomando o limite $\mathrm{r} \rightarrow \mathrm{R}$ em (15), que nos dá o desenvolvimento explicitado na Equação (16)

$$
\lim _{\mathrm{r} \rightarrow \mathrm{R}} \frac{\pi\left(\mathrm{P}_{1}-\mathrm{P}_{2}\right)}{2 \eta \mathrm{l}} \cdot\left(\frac{\mathrm{R}^{2} \mathrm{r}^{2}}{2}-\frac{\mathrm{r}^{4}}{4}\right)=\frac{\pi\left(\mathrm{P}_{1}-\mathrm{P}_{2}\right)}{8 \eta \mathrm{l}} \mathrm{R}^{4} .
$$

O resultado obtido pela Equação (15) é a lei de Poiseuille apresentada na Equação (1), com o detalhe de estarmos explicitando o gradiente de pressão $\Delta \mathrm{P}$ por $\mathrm{P}_{1}-\mathrm{P}_{2}$. É interessante discutirmos esse resultado, em especial as considerações tomadas para sua dedução. Em especial, a Equação (14) mostra a obtenção da integral partindo de um limite de somas infinitas além de que os teoremas 1 e 2 - que são a base para a conexão dos conceitos entre derivadas e integrais - aparecem fortemente ao passo que conectam a modelagem em torno da variação de fluxo sanguíneo em um anel com a vazão total $\mathrm{V}$.

Ainda nesse contexto, torna-se imperativo analisarmos a lei de Poseuille no que tange aos termos que a compõem, de modo que possamos obter informações relevantes do comportamento do fluxo sanguíneo. Com esse objetivo, reescreveremos essa lei através da Equação (17)

$$
\mathrm{V}=\beta \frac{\Delta \mathrm{P}}{\mathrm{l}}, \quad \beta=\frac{\pi \mathrm{R}^{4}}{8 \eta}
$$

A escrita da Equação (17) explicita os termos de pressão e do comprimento 1, visto que com isso podemos estabelecer a relação em que a vazão é proporcional a uma queda de pressão por unidade de comprimento 1 em termos de uma constante $\beta$. Essa colocação torna-se interessante devido ao caráter físico do problema modelado, mostrando que para maiores valores do comprimento 1 teremos uma menor vazão, ou seja, um menor fluxo sanguíneo.

\subsection{Um modelo para vasos obstruídos}

Com a lei de Poiseuille já deduzida podemos utilizá-la para modelar o fluxo sanguíneo sob algumas condições biológicas, em especial para o caso de obstruções dos vasos sanguíneos que acarretam 
variações nas pressões. Em especial, partiremos dos modelos propostos por [4] e então o generalizaremos para obtermos uma lei de Poiseuille modificada para cada um dos casos. De início, analisaremos um capilar que sofre obstrução, como apresentado na Figura 6.

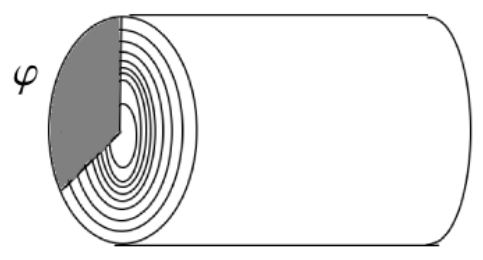

Figura 6: Esquematização para o modelo de obstrução em um capilar por um arco de circunferência.

Esse modelo apresenta a obstrução sob a hipótese de que a mesma possa ser aproximada por um arco de circunferência com ângulo central $\varphi$. Logo, a diminuição da área do anel circular dá-se pela área desse setor circular que é $\frac{\varphi}{2 \pi}\left(\pi \mathrm{r}_{\mathrm{i}}^{2}\right) \operatorname{com} \varphi \in(0,2 \pi)$, em que os $\mathrm{r}_{\mathrm{i}}$ são os mesmos esquematizados na Figura 5. Assim, podemos partir da Equação (13) para a vazão $\mathrm{V}_{\varphi}$ que considera apenas porção não obstruída do anel circular dada pela Equação (18)

$$
\Delta \mathrm{V}=\sum_{\mathrm{i}=1}^{\mathrm{n}}\left(1-\frac{\varphi}{2 \pi}\right)\left[2 \pi \mathrm{r}_{\mathrm{i}} \Delta \mathrm{r}_{\mathrm{i}} \cdot \frac{\left(\mathrm{P}_{1}-\mathrm{P}_{2}\right)}{4 \eta \mathrm{l}}\left(\mathrm{R}^{2}-\mathrm{r}_{\mathrm{i}}^{2}\right)\right]
$$

Com isso, obtemos, de forma análoga ao feito na subseção anterior, uma variação de lei de Poiseuille, descrita pela Equação (19)

$$
\mathrm{V}_{\varphi}=\left(1-\frac{\varphi}{2 \pi}\right) \beta \frac{\Delta \mathrm{P}_{\varphi}}{\mathrm{l}}, \quad \beta=\frac{\pi \mathrm{R}^{4}}{8 \eta}
$$

Além de modelarmos a obstrução por uma área de um arco de circunferência dos anéis dos capilares, podemos ainda explorar uma variação do raio $\mathrm{R}$ como feito por [4]. Isto é, consideraremos um caso em que o raio dos anéis terão máximo comprimento dado por $\phi$ como mostrado na Figura 7.

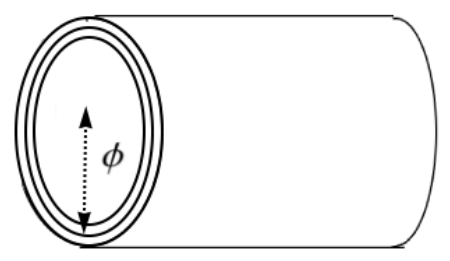

Figura 7: Esquematização para o modelo de obstrução através de um novo raio $\phi<\mathrm{R}$.

Nesse caso, precisaremos garantir que $\phi<\mathrm{R}$, para tanto tomaremos que $\phi=\gamma \mathrm{R}$, em que $\gamma$ é uma constante positiva tal que $\gamma \in(0,1)$. Partindo disso, podemos obter uma lei de Poiseuille para esse modelo, em que basta aplicarmos diretamente a condição do raio $\phi$ em (17), de modo que temos a vazão $\mathrm{V}_{\phi}$ dada na Equação (20) 


$$
\mathrm{V}_{\phi}=\gamma^{4} \beta \frac{\Delta \mathrm{P}_{\phi}}{\mathrm{l}}, \quad \beta=\frac{\pi \mathrm{R}^{4}}{8 \eta}
$$

Esses dois modelos de obstrução são interessantes de serem obtidos, pois permitem um modelo com certa generalidade para os capilares. Ademais, podemos ainda estabelecer uma relação entre o fluxo $\mathrm{V}$ da lei de Poiseuille usual, e suas modificações $\mathrm{V}_{\varphi}$ e $\mathrm{V}_{\phi}$ através de suas razões, as quais são apresentadas na Equação (21)

$$
\left\{\begin{array}{l}
\mathrm{V}_{\varphi}=\left(1-\frac{\varphi}{2 \pi}\right) \frac{\Delta \mathrm{P}_{\varphi}}{\Delta \mathrm{P}} \mathrm{V} \\
\mathrm{V}_{\phi}=\gamma^{4} \frac{\Delta \mathrm{P}_{\phi}}{\Delta \mathrm{P}} \mathrm{V}
\end{array}\right.
$$

Em singular, esta subseção possui um valor interesse para o trabalho no aspecto da modelagem, visto que, ambos os modelos obtidos são feitos com base na lei de Poiseuille e utilizando aspectos geométricos do próprio modelo. Não só isso, mas as mesmas ressaltam a concordância com os modelos já obtidos, dado que no limite dos modelos $\varphi \rightarrow 2 \pi$ e $\gamma \rightarrow 1$ restauramos a lei de Poiseuille.

\subsection{Um modelo para ramificação de vasos}

De posse da lei de Poiseuille podemos ainda expandir o modelo para circulação sanguínea através de determinar o ângulo ótimo $\theta \in(0, \pi / 2)$ para a ramificação de vasos sanguíneos; isto é, o ângulo que favoreça o escoamento sanguíneo ao longo dos capilares. Para isso, começaremos destacando a Figura 8-(a) como uma esquematização dos vasos sanguíneos, além de que consideraremos que a ramificação ocorre para vasos de menor raio, então: $r_{1}>r_{2}$ e na região da ramificação teremos um modelo que será aproximado para a representação da Figura 8-(b).

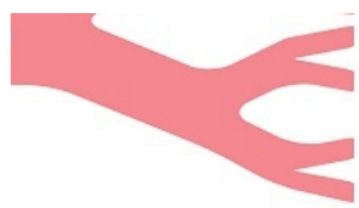

(a)

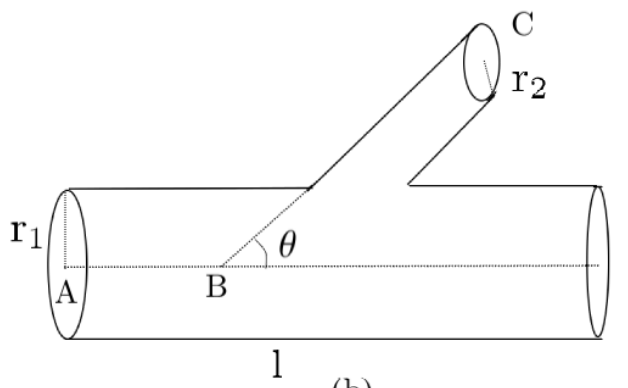

(b)

Figura 8: Em (a) representação do sistema arterial e em (b) modelo para estudo da ramificação vascular.

Não obstante, devemos, ainda, definir uma nova variável de interesse: a resistência do fluido. De fato, o processo de escoamento não é ideal; isto é, há várias condições do próprio sistema que corroboram para isso, tais como: irregularidades na trajetória ou a rispidez dos vasos. Por conta 
disso, é necessário definir um termo que descreva esse aspecto do sistema, o qual denominaremos por resistência do sistema, ou simplesmente resistência e denotado por $\mathrm{R}^{\mathrm{l}}$.

Por conseguinte, explicitaremos a resistência $\mathrm{R}^{\mathrm{l}}$ em termos das variáveis já definidas em nosso modelo da lei de Poiseuille. A ideia para isso é observarmos que a resistência $\mathrm{R}^{1}$ é proporcional ao gradiente da pressão $\Delta \mathrm{P}$ e inversamente proporcional ao fluxo $\mathrm{V}$ do fluido.

Essa discussão teórica é feita de modo a entendermos como deve ser o comportamento do termo de resistência. Entendemos, através do modelo teórico, uma possível expressão para o mesmo, ou permitindo que tenhamos uma noção do seu comportamento. No entanto, esse termo foi determinado de modo empírico por Poiseuille, dado por $\mathrm{R}^{1}=\frac{\Delta \mathrm{P}}{\mathrm{V}}$. Aqui, recorreremos ao resultado obtido empiricamente, mas sem abandonarmos as ideias de um modelo teórico, visto que, partindo do dado real realizaremos a descrição referente ao ângulo ótimo já mencionada acima.

Ademais, destacamos ainda que o uso de tal resultado torna-se imprescindível para esse trabalho no que tange o uso da modelagem no ensino, visto que, proporcionamos não só o confronto, mas a união de resultados teóricos e experimentais, corroborando para associação da matemática ao mundo real, em que reconhece-se o objeto estudado e busca-se hipóteses de solução para o mesmo.

Efetuada essas considerações, podemos, agora, expressar a resistência na lei de Poiseuille, em que usando $\mathrm{R}^{1}=\frac{\Delta \mathrm{P}}{\mathrm{V}}$ na Equação (16) e realizando algumas manipulações algébricas tem-se o resultado explicitado na Equação (22)

$$
\mathrm{R}^{\mathrm{l}}=\mathrm{M} \frac{\mathrm{l}}{\mathrm{R}^{4}} .
$$

O termo $\mathrm{M}$ é uma nova constante associada à viscosidade do fluido, dada por: $\mathrm{M}=\frac{8 \eta}{\pi}$. Notemos que a resistência $\mathrm{R}^{1}$ é proporcional ao comprimento do cilindro, visto que para um maior cilindro o fluido terá mais que escoar por uma trajetória mais longa, mas a mesma é inversamente proporcional ao raio elevado à quarta potência, mostrando que a expansão do raio $\mathrm{R}$ diminui a resistência do escoamento de forma quártica.

Definido essa grandeza, podemos enfim modelar nosso problema para a determinação do ângulo ótimo. Como explicitado na Equação (22), temos o destaque para o comprimento l e o raio R, os quais serão determinados tendo em vista a geometria presente na Figura (8), à qual daremos maior ênfase num modelo simplificado apresentado na Figura 9.

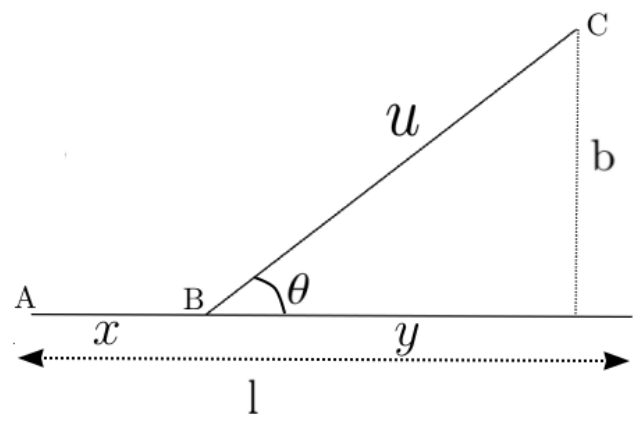

Figura 9: Esquematização dos aspectos geométricos do modelo para ramificações da Figura 8. 
Nosso objetivo, agora, será explicitar alguns termos presentes na esquematização do nosso modelo na Figura 9, de modo que esses nos auxiliem na determinação do ângulo ótimo $\theta$. Então, explorando os aspectos geométricos e trigonométricos da Figura 9 temos as relações da Equação (23)

$$
\left\{\begin{array}{l}
\mathrm{y}=\mathrm{b} \cot (\theta), \\
\mathrm{x}=\mathrm{l}-\mathrm{b} \cot (\theta), \\
\mathrm{u}=\mathrm{b} \csc (\theta) .
\end{array}\right.
$$

Como já mencionado, buscaremos através da Equação (22) descrever o circuito $\mathrm{ABC}$, em que consideraremos esse circuito como sendo a soma dos circuitos $A B$ e $B C$, de forma que a $R_{A B C}$ seja a soma das resistências das duas partes do circuito. Efetuaremos esse tratamento de modo a obtermos uma descrição efetiva para o modelo proposto na Figura 8, visto que o circuito BC possui um raio $\mathrm{r}_{2}$, diferente do raio $\mathrm{r}_{1}$ do circuito $\mathrm{AB}$. Assim, podemos, com auxílio das Equações (22) e (23), descrever a resistência total como:

$$
\begin{aligned}
\mathrm{R}_{\mathrm{ABC}}(\theta) & =\mathrm{R}_{\mathrm{AB}}+\mathrm{R}_{\mathrm{BC}} \\
& =\mathrm{M}\left[\frac{1-\mathrm{b} \cot (\theta)}{\mathrm{r}_{1}^{4}}\right]+\mathrm{M}\left[\frac{\mathrm{b} \csc (\theta)}{\mathrm{r}_{2}^{4}}\right] \\
& =\mathrm{M}\left[\frac{1-\mathrm{b} \cot (\theta)}{\mathrm{r}_{1}^{4}}+\frac{\mathrm{b} \csc (\theta)}{\mathrm{r}_{2}^{4}}\right] .
\end{aligned}
$$

A formulação obtida em (24) trata a resistência não como uma equação, mas sim como uma função da variável $\theta$. Com essa função, encontraremos o ângulo ótimo e para isso abordaremos essa questão como sendo um problema de máximos e mínimos de uma função de uma variável em que determinaremos os pontos críticos da função e verificaremos aqueles que tornam a resistência mínima, visto que isso acarreta maior escoamento possível para o sangue. Para isso, é necessário definirmos alguns conceitos e proposições conforme colocado por $[6,8]$ para essa abordagem.

Definição 2. Um ponto crítico de uma função $\mathrm{f}(\mathrm{x})$ é um número c no domínio da função tal que $\mathrm{f}^{\prime}(\mathrm{c})=0$ ou $\mathrm{f}^{\prime}(\mathrm{c})$ não existe.

Definição 3. Um número f(c) é um valor de

(a) Máximo local quando $f(c) \geq f(x)$ quando $x$ está próximo de c;

(b) Mínimo local quando $\mathrm{f}(\mathrm{c}) \leq \mathrm{f}(\mathrm{x})$ quando $\mathrm{x}$ está próximo de c.

Teorema 3. (Teorema de Fermat) Se uma função $\mathrm{f}(\mathrm{x})$ tiver um máximo ou mínimo local em c $e$ se $\mathrm{f}^{\prime}(\mathrm{c})$ existir, então $\mathrm{f}^{\prime}(\mathrm{c})=0$.

Demonstração. Ver referência [6].

As definições 2 e 3 introduzem conceitos fundamentais no estudo de funções. Além disso, o Teorema de Fermat (Teorema 3) estabelece uma relação entre as duas definições, de modo que um ponto de máximo ou mínimo local esteja atrelado à existência de um ponto crítico c. Com isso explicitado, mostraremos que a função (24) admite um ponto crítico. Para isso calcularemos a derivada da função que será explicitada na Equação (25) 


$$
\frac{\mathrm{dR}_{\mathrm{ABC}}}{\mathrm{d} \theta}=\mathrm{M}\left[\frac{\mathrm{b} \csc ^{2}(\theta)}{\mathrm{r}_{1}^{4}}-\frac{\mathrm{b} \cot (\theta) \csc (\theta)}{\mathrm{r}_{2}^{4}}\right]
$$

Com isso, seguiremos na determinação do ponto crítico, buscando um ponto c para qual a Equação (25) seja identicamente nula, o que nos leva ao seguinte desenvolvimento:

$$
\begin{aligned}
\mathrm{M}\left[\frac{\mathrm{b} \csc ^{2}(\theta)}{\mathrm{r}_{1}^{4}}-\frac{\mathrm{b} \cot (\theta) \csc (\theta)}{\mathrm{r}_{2}^{4}}\right] & =0 \\
\frac{\csc ^{2}(\theta)}{\mathrm{r}_{1}^{4}}-\frac{\cot (\theta) \csc (\theta)}{\mathrm{r}_{2}^{4}} & =0 \\
\frac{1}{\mathrm{r}^{4}}-\frac{\cos (\theta)}{\mathrm{r}_{2}^{4}} & =0 \\
\therefore \cos (\theta) & =\left(\frac{\mathrm{r}_{2}}{\mathrm{r}_{1}}\right)^{4} .
\end{aligned}
$$

O resultado obtido na Equação (26) é o ponto crítico associado à Função (24). Aqui, destacamos que também podemos explicitar o ponto crítico diretamente em termos de $\theta$, com uso da função inversa do cosseno.

Nesse sentido, é oportuno ressaltarmos que esse resultado assegura-nos a existência de um ponto crítico associado à função do nosso modelo, porém não garante a existência de máximos e mínimos locais. Isto é, as definições 2, 3 bem como o Teorema 3 não nos mostram como encontrar esses valores, mas, sim, mostram-nos que o ponto crítico associado à função é um candidato a um desses valores. Nesse sentido, podemos ainda recorrer a duas proposições presentes no cálculo diferencial que permitirão a classificação, ou não, do ponto crítico em máximo ou mínimo local.

Proposição 1. (Teste da derivada primeira) Suponha que c seja um ponto crítico de uma função contínua $\mathrm{f}(\mathrm{x})$. Então têm-se as seguintes possibilidades,

(a) Se o sinal de $\mathrm{f}^{\prime}(\mathrm{x})$ mudar de positivo para negativo em c, então $\mathrm{f}(\mathrm{x})$ tem um máximo local em $\mathrm{c}$;

(b) Se o sinal de $\mathrm{f}^{\prime}(\mathrm{x})$ mudar de negativo para positivo em c, então $\mathrm{f}(\mathrm{x})$ tem um mínimo local em c;

(c) Se o sinal de $\mathrm{f}^{\prime}(\mathrm{x})$ não mudar em c, então $\mathrm{f}(\mathrm{x})$ não possui máximos ou mínimos locais em $\mathrm{c}$.

Demonstração. Ver referência [6].

Proposição 2. (Teste da derivada segunda) Suponha que $f$ seja contínua na proximidade do ponto crítico c. Então têm-se as seguintes possibilidades

(a) Se $\mathrm{f}^{\prime}(\mathrm{c})=0$ e $\mathrm{f}^{\prime \prime}(\mathrm{c})>0$ então $\mathrm{f}(\mathrm{x})$ possui um ponto de minimo local em $\mathrm{c}$;

(b) Se $\mathrm{f}^{\prime}(\mathrm{c})=0$ e $\mathrm{f}^{\prime \prime}(\mathrm{c})<0$ então $\mathrm{f}(\mathrm{x})$ possui um ponto de máximo local em $\mathrm{c}$. 
Demonstração. Ver referência [6].

Ambas as proposições 1 e 2 permitem a avaliação de um ponto crítico, desde que suas hipóteses sejam satisfeitas. Ao analisarmos a Função (25) é perceptível uma descontinuidade em $\theta=0$, com isso não podemos utilizar a proposição 1 para classificação do ponto dado em (26). Entretanto, a proposição 2 tem por hipótese uma continuidade definida localmente em termos do ponto crítico. Assim, podemos nos valer da mesma para a classificação do ponto, de modo que a derivada segunda da Função (24) será dada pela Função (27)

$$
\frac{\mathrm{d}^{2} \mathrm{R}_{\mathrm{ABC}}}{\mathrm{d} \theta^{2}}=\mathrm{M}\left[\frac{\mathrm{b} \cot ^{2}(\theta) \sec (\theta)+\mathrm{b} \csc ^{3}(\theta)}{\mathrm{r}_{2}^{4}}-\frac{2 \mathrm{~b} \csc ^{2}(\theta) \cot (\theta)}{\mathrm{r}_{1}^{4}}\right] .
$$

Agora, verificaremos o sinal da Função (27) com (26), que nos leva ao seguinte desenvolvimento,

$$
\begin{aligned}
\left.\frac{\mathrm{d}^{2} \mathrm{R}_{\mathrm{ABC}}}{\mathrm{d} \theta^{2}}\right|_{\cos (\theta)=\left(\frac{\mathrm{r}_{2}}{\mathrm{r}_{1}}\right)^{4}} & =\mathrm{Mb}\left[\frac{\cot ^{2}(\theta) \csc (\theta)+\csc ^{3}(\theta)}{\mathrm{r}_{2}^{4}}-\frac{2 \csc ^{2}(\theta) \cot (\theta)}{\mathrm{r}_{1}^{4}}\right] \\
& =\frac{\mathrm{Mb}}{\operatorname{sen}^{3}(\theta)}\left[\frac{\cos ^{2}(\theta)}{\mathrm{r}_{2}^{4}}+\frac{1}{\mathrm{r}_{2}^{4}}-\frac{2 \cos (\theta)}{\mathrm{r}_{1}^{4}}\right] \\
& =\frac{\mathrm{Mb}}{\operatorname{sen}^{3}\left(\arccos \left(\mathrm{r}_{2}^{4} / \mathrm{r}_{1}^{4}\right)\right)}\left[\frac{\mathrm{r}_{2}^{4}}{\mathrm{r}_{1}^{8}}+\frac{1}{\mathrm{r}_{2}^{4}}-\frac{2 \mathrm{r}_{2}^{4}}{\mathrm{r}_{1}^{8}}\right] \\
& =\frac{\mathrm{Mb}}{\operatorname{sen}^{3}\left(\arccos \left(\mathrm{r}_{2}^{4} / \mathrm{r}_{1}^{4}\right)\right)}\left[\frac{\mathrm{r}_{1}^{8}-\mathrm{r}_{2}^{8}}{\mathrm{r}_{1}^{8} \mathrm{r}_{2}^{8}}\right] .
\end{aligned}
$$

De acordo com nossas hipóteses de trabalho, temos que $\theta$ é um ângulo do primeiro quadrante e $\operatorname{logo} \operatorname{sen}(\theta)>0$. Além disso, como $r_{1}>r_{2}$ temos então que $r_{1}^{8}-r_{2}^{8}>0$. Com isso, a Equação (28) é maior que zero para todo $\theta$, portanto, pelo teste da derivada segunda (Proposição 2) o ponto encontrado em (26) é um ponto de mínimo, o que permite concluirmos que o ângulo ótimo é de fato obtido por essa equação e pode ser dado explicitamente pela Equação (29)

$$
\theta=\arccos \left(\frac{\mathrm{r}_{2}}{\mathrm{r}_{1}}\right)^{4}
$$

Como mostramos que o ponto crítico é um mínimo local, então ele minimiza a função da resistência, de modo que quando minimizada por (26) é expressa na Equação (30)

$$
\mathrm{R}_{\mathrm{ABC}}=\mathrm{M}\left[\frac{\mathrm{l}}{\mathrm{r}_{1}^{4}}-\frac{\mathrm{br}_{2}^{4}}{\mathrm{r}_{1}^{8} \sqrt{1-\mathrm{r}_{2}^{4} / \mathrm{r}_{1}^{4}}}+\frac{\mathrm{b}}{\mathrm{r}_{2}^{4} \sqrt{1-\mathrm{r}_{2}^{4} / \mathrm{r}_{1}^{4}}}\right] \text {. }
$$

Nesta subseção modelamos o comportamento da resistência do escoamento sanguíneo ao longo de vasos que se ramificam. Aqui, destacamos a forte conexão dos aspectos fundamentais da modelagem que corroboram para a inserção dos conceitos do CDI, em singular dos problemas de otimização 
e do estudo de máximos e mínimos de funções objetivando a caracterização do ângulo ótimo do escoamento, bem como minimizando a resistência do escoamento. Outrossim, por meio da reformulação da lei de Poiseuille para esse modelo, temos também a garantia de um contraexemplo à proposição 1, que se torna de grande relevância, visto que associa ao problema um aspecto fundamental presente no CDI: a continuidade de funções.

\section{Algumas considerações}

Com as ideias aqui abordadas, objetivando reflexões e compreensões acerca de formas de abordamos conceitos do Cálculo Diferencial e Integral (CDI) usando a Lei de Poiseuille via Modelagem Matemática, evidenciamos diferentes meios que podem ser usados pelo professor no ambiente de sala de aula no processo de ensino e aprendizagem da Matemática.

Neste artigo tivemos especial interesse em apresentar uma alternativa de ensino que provoque reflexões e motive o ensino e a aprendizagem de CDI. O artigo visa encaminhar uma reflexão sobre a ideia de que há alguns aspectos cuja inserção às aulas pode provocar contribuições significativas para a aprendizagem dos alunos.

Evidenciamos que, geralmente, um aspecto importante é trabalhar com problematizações e desenvolver no âmbito da sala de aula atividades que provoquem os alunos a mobilizarem o seu conhecimento e a perceberem aplicações desse em situações do mundo real. Neste movimento abordamos a modelagem matemática como uma alternativa de ensino e aprendizagem para as aulas de CDI.

Exploramos algumas situações, tais como a circulação sanguínea, nas quais abordagens com modelagem foram desenvolvidas. O que se pode concluir é que, por um lado, pensar nas atividades das aulas de CDI como algo que envolve ideias e apresenta sentido no mundo real para os alunos pode ser fator importante para a aprendizagem e, desse modo, podendo contribuir para dirimir os problemas que se percebem em relação ao ensino e à aprendizagem nessa disciplina nos cursos de graduação.

Por fim, destacamos ainda que a lei de Poiseuille foi desenvolvida e apresentada para o caso específico da circulação sanguínea. Todavia, dadas as hipóteses de trabalho por nós consideradas, a mesma apresenta aplicabilidade para uma longa classe de fluidos, como os viscosos; a exemplo do mel, óleo e o petróleo. Dessa forma, permite que a mesma seja estendida para outras situações cotidianas que também podem ser de grande valor para o ensino do CDI em sala de aula.

Com isso entendemos que não devemos pensar a Matemática consistindo apenas em cálculos, sem lugar para o espírito criativo, investigativo e motivador.

\section{Referências}

[1] Bassanezi, Rodney Carlos. Ensino-aprendizagem com Modelagem Matemática. $3^{\text {a }}$ edição; São Paulo: Contexto, 2011.

[2] Batschelet, E., Introdução à Matemática para Biocentistas. São Paulo: EdUSP, 1978.

[3] Eves, Howard. Introdução à História da Matemática. Campinas: Unicamp, 2004.

[4] Fluxo Sanguíneo: Uma Aplicação da Integral de Riemann. 2007. Disponível em: <http:// www.portal.famat.ufu.br/sites/famat.ufu.br/files/Anexos/Bookpage/Fa-mat_revista_09_sala_04.pdf > .

Acesso em 12 de maio de 2021. 
[5] Gondar, Juan López; Cipolatti, Rolcide Almeida. Iniciação à Física Matemática: modelagem de processos e métodos de solução. Rio de Janeiro: Impa, 2009.

[6] Guidorizzi, H. L. Um curso de Cálculo, vol 1. 6º ed. Editora LTC, 2008.

[7] Nussenzveig, H. Moyses. Curso de Física Básica 2-Fluidos, Oscilações e Ondas, Calor. São Paulo: Editora Edgard Blücher Ltda, 2002.

[8] Stewart, James. Calculus: early transcendentals. Boston: Cengage Learning, 2016.

[9] Sutera SP, Skalak R. 1993. ' The history of Poiseuille's law." Annu. Rev. Fluid Mech.25:1-19

[10] Táboas, P.Z. Cálculo em uma Variável. São Paulo: EdUSp, 2008.

[11] Peres, R. Novas tendências no ensino de cálculo, as acompanhamos?. Trabalho apresentado no XXXIX CNMAC, Uberlândia - MG, 2019. Anais.

Gabriel Fernandez Ferrari Melo

Universidade Estadual da Região Tocantina do Maranhão Centro de Ciências Exatas, Naturais e Tecnológicas (CCENT) <gabriel.melo@uemasul.edu.br>

Juscimar da Silva Araujo

Universidade Estadual da Região Tocantina do Maranhão Centro de Ciências Exatas, Naturais e Tecnológicas (CCENT)

$<$ juscimararaujo@uemasul.edu.br>

Recebido: 04/06/2021

Publicado: 14/02/2022 